\title{
FUSION PROGRAMS IN APPLIED PLASMA PHYSICS
}

OCTOBER 1, 1991 THROUGH JANUARY 31, 1992 CONTRACT EXTENSION REPORT

by

PROJECT STAFF

\author{
Work prepared under \\ Department of Energy \\ Contract DE-AC03-89ER53277
}

GENERAL ATOMICS PROJECT 3468 DATE PUBLISHED: JULY 1992

\section{GENERAL ATOMIES




\section{DISCLAIMER}

This report was prepared as an account of work sponsored by an agency of the United States Government. Neither the United States Government nor any agency thereof, nor any of their employees, makes any warranty, express or implied, or assumes any legal liability or responsibility for the accuracy, completeness, or usefulness of any information, apparatus, product, or process disclosed, or represents that its use would not infringe privately owned rights. Reference herein to any specific commercial product, process, or service by trade name, trademark, manufacturer, or otherwise, does not necessarily constitute or imply its endorsement, recorrumendation, or fayoring by the United States Government or any agency thereof. The views and opinions of authors expressed herein do not necessarily state or reflect those of the United States Government or any agency thereof.

This report has been reproduced directly from the best available copy

Available to DOE and DOE contractars from the Office of Scientrific and' Techunical Information P.O. Box 62

Oak Ridge, TN 37331

Prices available from (315) 576-8401, Frs 628-8401

Available to the public from the National Technical Information Service

U.S. Department of Commerce 5285 Part Royal Rosd Sprinugfield, VA 22161 


\section{CONTENTS}

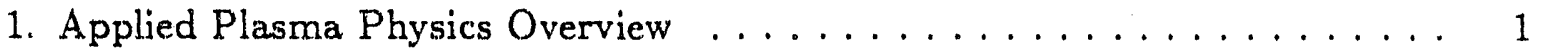

2. Applied Plasma Physics Theory Program . . . . . . . . . . . . . 2

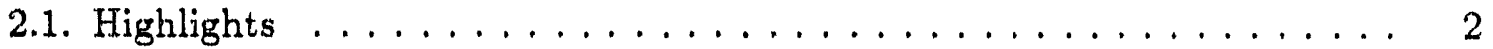

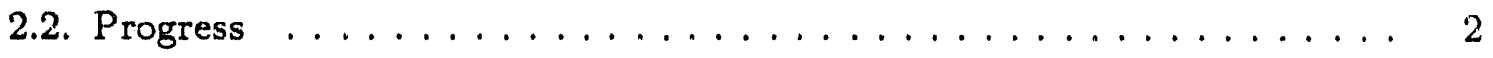

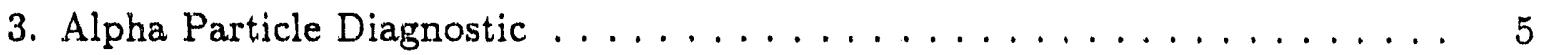

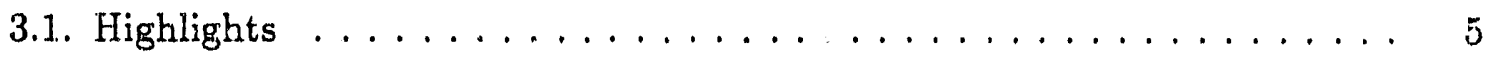

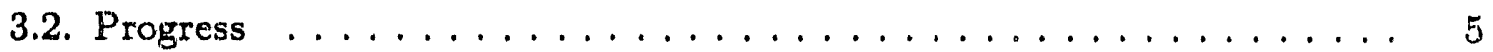

4. Edge and Current Density Diagnostic ............... 8

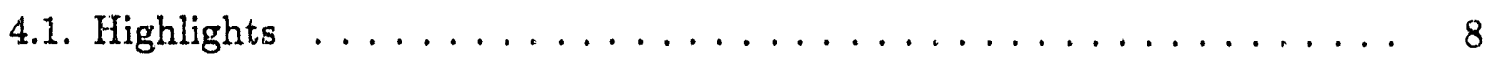

4.2. Progress $\ldots \ldots \ldots \ldots \ldots \ldots \ldots \ldots \ldots \ldots \ldots \ldots \ldots \ldots \ldots \ldots$

5. Fusion User Service Center $\ldots \ldots \ldots \ldots \ldots \ldots$

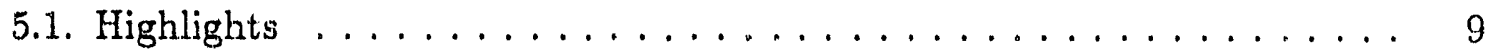

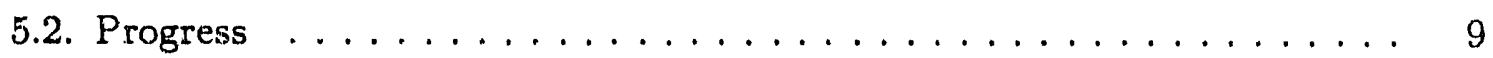

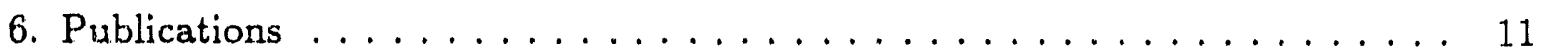

\section{FIGURES}

1. Schematic of simple neutral particle detector used on TFTR . . . . . . 6

2. Scintillation light pattern observed during lithium pellet injection into a deuterium beam-heated TFTR discharge $\ldots \ldots \ldots \ldots \ldots$ 


\section{APPLIED PLASMA PHYSICS OVERVIEW}

The Applied Plasma Physics (APP) program at General Atomics (GA) described here includes four major elernents: (a) Applied Plasma Physics Theory Program, (b) Alpha Particle Diagnostic, (c) Edge and Current Density Diagnostic, and (d) Fusion User Service Center (USC).

The objective of the APP theoretical plasma physics research at GA is to support the DIII-D and other tokamak experiments and to significantly advance our ability to design a commercially-attractive fusion reactor. We categorize our efforts in three areas: magnet,ohydrodynamic (MHD) equilibria and stability; plasma transport with emphasis on $\mathrm{H}$-mode, divertor, and boundary physics; and radio frequency ( $\mathrm{r} f$ ).

The objective of the APP alpha particle diagnostic is to develop diagnostics of fast confined alpha particles using the interactions with the ablation cloud surrounding injected pellets and to develop diagnostic systems for reacting and ignited plasmas.

The objective of the APP edge and current density diagnostic is to first develop a lithium beam diagnostic system for edge fluctuation studies on the Texas Experimental Tokamak (TEXT).

The objective of the Fusion USC is to continue to provide maintenance and programming support to computer users in the GA fusion community.

The detailed progress of each separate program covered in this report period is described in the following sections. 


\section{APPLIED PLASMA PHYSICS THEORY PROGRAM}

\subsection{HIGHLIGHTS}

- Thirteen papers with theory group members as authors or coauthors were presented at the 33rd Annual Meeting of the Division of Plasma Physics, American Physical Society held November 4-8, 1991 at Tampa, Florida.

- 'The DOE Office of Program Assessment held a review of the Applied Plasma Physics (APP) program (January 21-22, 1992, La Jolla) which included the CQL3D Fokker-Planck code. It was well received. W. Sadowski, Branch Chief of DOE/APP Theory, visited GA on January 24 and was briefed on the theory progress and plan for this year.

\subsection{PROGRESS}

A visit by $\mathrm{H}$. Berk from the Institute for Fusion Studies to collaborate on the continuum damping of Toroidal Alfvén Eigenmodes (TAE) has helped clarify a few theoretical points and contributed to our progress on understanding and modeling of continuum damping. Subsequently, a method has been found to compute the continuum damping for TAE modes. The method is a generalization of that used for tearing modes. The method produces damping rates, hence stability threshold values. It is being tested on model analytic problems posed by Rosenbluth et al. If the test is successful, the method can be incorporated in the GATO code.

The possibility of using of to influence TAE modes was looked at. Within the parameter space in which TAE modes were observed in DIII-D, there is a possibility of using fast waves to heat either the ions or the electrons through appropriate antenna phasing, and thus influence the stability of TAE modes. Experimentally, this can be tested by bringing the plasma to a marginal state by neutral beam injection (NBI) and then applying the of power.

Work has begun on including an option for the slow sound approxirnation in the GATO stability code. This will eliminate the acousic continua, which is a major source of difficulty in calculating Alfvén modes in real geometry, but still retain. approximately the correct frequency dependence of these modes. 
An improved version of the paper on tearing mode energy principle was completed. It is found that in some cases when the configuration is ideally unstable, the formalism will predict the instability but may not be accurate in calculating the mode structure. The reason is und $r$ investigation.

General Atomics Report GA-A20629, Turbulent Viscosity Bifurcation and the H-Mode, by G.M. Staebler and R.R. Dominguez, was issued. In this report the quasilinear viscous stress due to kinetic ion temperature gradient (ITG) modes in a sheared slab is shown to possess a maximum at a critical value for the gradient of the equilibrium electric field. Combining ITG mode and dissipative trapped electron mode contributions, a classic bifurcation curve for the turbulent viscous stress as a function of the $E \times B$ flow shear is found. The L-mode of tokamaks is similar to the low flow shear solution in which the ITG mode is unstable. The H-mode naturally corresponds to the high flow shear solution in which the ITG mode is linearly stable.

Work continued on the implementation of the gyro-Landau fluid model in the $3 \mathrm{D}$ TRB code. The new numerical methods are able to find toroidal ion temperature gradient ballooning modes, but saturated turbulence runs in toroidal geometry have yet to work. The UNIX version of the TRB turbulence code has been modified with new numerical methods to properly evolve off-helicity modes and updated to handle the new gyro-Landau fluid toroidal terms.

New work was initiated to study stability of microturbulence in the second stability regime. This began with a literature survey.

General Atomics Report GA-A20343, On the Universal Toroidal Ohm's Law for Tokamaks, by R.O. Dendy and R.W. Harvey was issued. This report discusses the simple formulation of the neoclassical toroidal Ohm's law for tokamaks previously given by A.A. Ware [Nucl. Fusion 13 (1973) 793]: $j_{\phi}=\sigma_{\text {Spitzer }}\left(E_{\phi}+V_{\mathrm{r}} B_{\theta}+\alpha \tilde{Q}_{\mathrm{r}} B_{\theta} /\right.$ $n T_{\mathrm{e}}$ ), where $V_{\mathrm{r}}$ and $\tilde{Q}_{\mathrm{r}}$ are the mean radial velocity and heat flux, and $\alpha$ is a numerical factor which depends weakly on ion charge. Perhaps surprisingly, it is valid for all collisionality regimes, depends on the Spitzer conductivity, and has no explicit dependence on the ratio of trapped to passing particles. This paper argues that the universal Ohm's law can be interpreted as a direct consequence of the principle of conservation of canonical toroidal angular momentum in toroidally symmetric systems.

A GA report $3 D$ Fokker-Planck Calculation of Combined Fast Wave/Lower Hybrid and Electron Current Drive in Tokamaks by R.W. Harvey, S.C. Chiu, M.G. McCoy, G.D. Kerbel, G.D. Smith, and T.K. Mau has been distributed and was sent to the publication committee of the International Atomic Energy Association (IAEA) 
Technical Cornmittee Meeting on Fast Wave Current Drive in Reactor Scale Tokamaks (September 23-25, 1991, Arles, France) for inclusion in the proceedings.

Studies with CQL3D rule out the possibility of a dc electric field/fast wave current drive (FWCD) synergy as an explanation of the observed FWCD being independent of launch direction of the of waves. Under conditions of the experiment in DIII-D, CQIJ3D indicates that the ohmic and rf-driven currents should simply be additive. The CQL3D Fokker-Planck code is now fully installed and functioning on the UNICOS Cray F-machine. The code computes electron cyclotron damping and current drive along with ray tracing. This portion of the code is being debugged.

A few upgrades and changes, including a bootstrap current calculation, dc electric field correction to current drift efficiency, and ability to input an externallygenerated antenna spectrum, were made in the ray-tracing code RAYLH.

In response to a request from the DOE's office of Program Analysis, an information package was prepared pursuant to the DOE/Computational Theory Program Research Review, La Jolla, January 21-23, 1992. 


\section{ALPHA PARTICLE DIAGNOSTIC}

\subsection{HIGHLIGHTS}

In the period October 1, 1991 through January 31, 1992, we completed installation of a simple neutral particle detector on the Tokamak Fusion Test Reactor (TFTR) and observed deuterium neutrals from beam-injected deuterium ions $(E \leqslant 100 \mathrm{keV})$ interacting with lithium pellet ablation clouds.

\subsection{PROGRESS}

The simple detector, shown in Fig. 1, consisted of a $250 \AA$-thick carbon foil to strip the escaping neutrals and a $15 \mu$-thick $\mathrm{ZnS}(\mathrm{Cu})$ scintillator to detect the resulting ions. The ions created in the stripping foil were bent downward using the 7 to $10 \mathrm{kG}$ residual toroidal magnetic field of the tokamak between toroidal field coils at the analyzer location. The low energy ions strike the end of the scintillator closest to the foil while the higher energy ions strike the scintillator further from the stripping foil. Using fiber optically coupled optics, the scintillation light was imaged onto a $\mathrm{X}$ ybion CID-003 gated image intensified TV camera.

The light pattern observed on the scintillator, shown in Fig. 2, was consistent with a signal due to beam-injected deuterium ions interacting with the pellet ablation cloud. The signal was only present during pellet injection. The scintillation pattern of Fig. 2 was for a discharge at full toroidal field. We also took data at low toroidal fields and that observed the signal moved to the high energy end of the scintillator as expected. The lower toroidal field bends the stripped deuterium ions more slowly so they strike the far end of the scintillator. If the observed scintillation light had been due to $x$-rays or light from the pellet cloud, we would not have observed this variation with toroidal field. A much weaker signal was observed with boron pellets and no signal was observed with carbon pellets as expected based on the variation in neutral equilibrium fractions for the clouds.

We have observed neutrals resulting from the interaction of neutral beaminjected deuterium ions interactive with the ablation cloud surrounding injected lithium pellets. The next step will be to use an energy-resolved neutral particle analyzer 


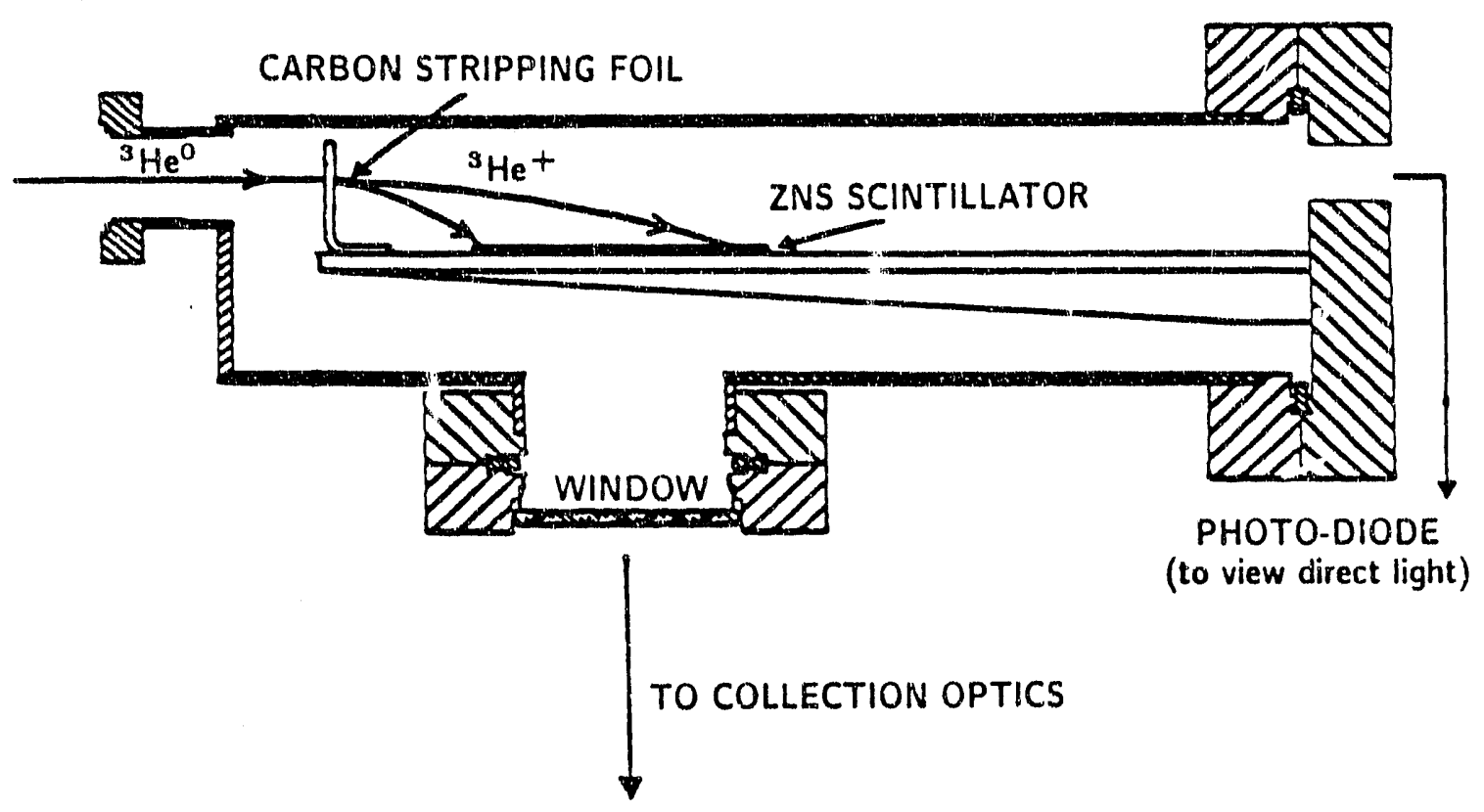

FIG. 1. Schematic of simple neutral particle detector used on TFTR. (Escaping neutrals incident from the left are imaged in a carbon stripping foil, bent downward by the residual tokamak toroidal field, and strike a $\mathrm{ZnS}$ scintillator. The light pattern on the scintillator is imaged onto a Xybion TV camera.)

to measure the energy spectrum of the neutrals produced as a result of cloud interactions and use this to calculate the energy spectrum of fast ions incident on the cloud. 


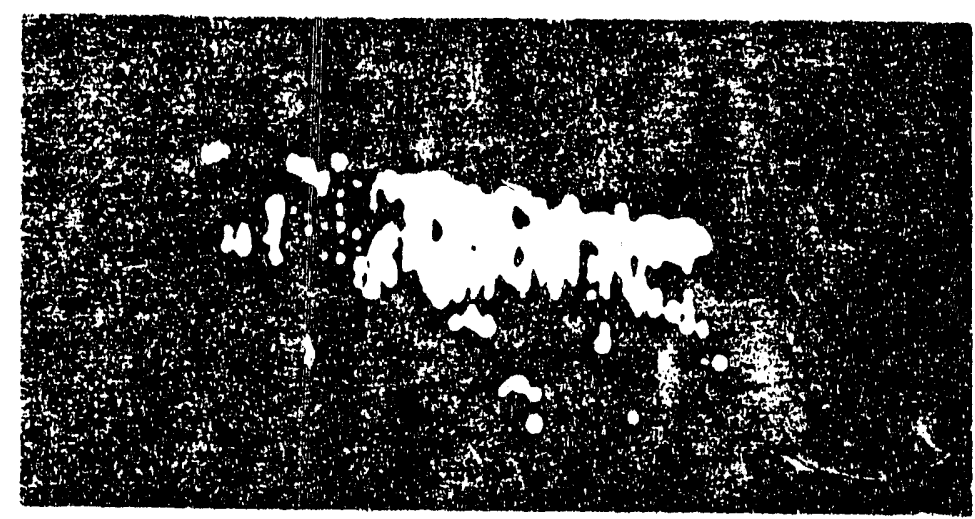

FIG. 2. Scintillation light pattern observed during lithium pellet injection into a deuterium bearn-heated TFTR discharge. This signal is corristent with observing deuterium neutrals resulting from the interaction of fast beam-injected ions interacting with the pellet ablation cloud. 


\section{EDGE AND CURRENT DENSITY DIAGNOSTIC}

\subsection{HIGHLIGHTS}

The lithium beam accelerator was re-assembled in Texas after being modified in San Diego. Magnetic shielding for the ion source and accelerator was fabricated and installed, and the accelerator was tested in the presence of the TEX'T magnetic field.

\subsection{PROGRESS}

A poster describing the diagnostic and the anticipated method of data analysis was presented at the 1991 Division of Plasma Physics Meeting, American Physical Society, in Tampa, Florida.

General Atomics personnel made two trips to Austin, Texas during this period. The lithium beam ion source, accelerator, neutralizer, vacuum system, and control electronics were re-assembled in Austin after having been modified in San Diego during the final quarter of FY91. Magnetic shielding for the ion source and beam line was fabricated in San Diego, shipped to Austin, and installed. The whole system was then tested in place in the tokamak laboratory, in the presence of the TEXT magnetic field. The main test consisted of measuring $\mathrm{Li}^{+}$current with a Faraday cup with and without the magnetic field. The beam current was monitored in the control room by the TEXT data acquisition system, and everything worked as expected.

Long overdue components for the second major subsystem of the diagnostic, the optical detection system, were finally received from the vendors. The mechanical design of the optical system was completed, and, with the receipt of the conıponents, fabrication was begun. 


\section{FUSION USER SERVICF CENTER}

\subsection{HIGHLIGHTS}

- The UNICOS conversion task made significant progress with the ONETWO Transport code nearly complete.

- Two HP9000/730 and one HP9000/720 workstations from Hewlett Packard (HP) and one Indigo workstation from Silicon Graphics (SGI) were procured and installed.

- The ESnet cisco router was upgraded twice and a Frame Relay circuit was installed and made operational.

- The VAX configuration change was completed; on-line disk storage was increased.

\subsection{PROGRESS}

Considerable progress was made in the UNICOS conversion task. The entire ONETWO transport code was moved to the new operating system. Most debugging and testing of the ONETWO Transport code was completed this period. In addition, the previously converted codes were upgraded to reflect the changes National Energy Research Supercomputer Center (NERSC) has made in the operating system, the Fortran compiler, and the math libraries. Other major codes converted or in the process of conversion are: MBC, GATO, TORAY, McGO. Temporary assistance on this project was provided by jobshoppers. In addition to code conversion, the USC continues to provide user training, consulting, documentation, and many software tools.

After a lengthy evaluation of available workstations, including benchmark testing, three workstations were purchased from HP and one from SGI. The HP workstations (two HP730 and a HP720) will be used for general physics code development and computations. The SGI Indigo will be used on the Fokker-Planck code CQL3D for $\mathrm{rf}$ and neutral beam heating simulations. The SGI workstation was operational in December 1991, the HP workstations were operational in January 1992. Additional memory (16MB each) was installed in the HP workstations. 
The ESnet cisco router was upgraded. A Frame Relay circuit was installed by Williams Telecommunications (WilTel) for NERSC. This circuit connects LLNL, GA, and PPPL, and has been installed for test and evaluation. A subsequent upgrade increased the capacity of the ESnet cisco router and included a FDDI interface. One of the VAX8650s was removed from the USC and installed at DIII-D. The VAX11/785 at DIII-D was returned to the USC where it will replace the VAX11/750. This swap of computers is related to the upgrade of the USC VAX cpu cycles last quarter of FY91 in which the VAX11/750 and a VAX11/780 were traded ont for two VAX4000300 s. The result of all of this is an increase in performance at both the USC and DIII-D. There were no major difficulties or problems with this swap. All systems continue to perform well.

Two System Industries (SI) disks were ordered for the VAX cluster, replacing two smaller, older RA81s. We received trade in credit for the RA81 disk drives. These will add about 1 Gbyte of space for storage of shot data and system files. The disks were installed and fully functional in January 1992.

All USC staff members attended part of Digital Equipment Corporation Users Society (DECUS) Symposium and the related equipment show, DEXPO. Both meetings were held in Anaheim, California. 


\section{PUBLICATIONS}

V.S. Chan and S.C. Chiu, Kinetic Aspects of Nonresonant Forces by RF Waves, Bull. Am. Phys. Soc. 36 (1991) 2339.

S.C. Chiu, T.K. Mau (UCLA), and R.W. Harvey, Theoretical Modeling of Fast Wave Current Drive in DIII-D, Bull. Am. Phys. Soc. 36 (1991) 2470.

M.S. Chu, L.L. Lao, A.D. Turnbull, and J.K. Lee, Toroidal Effect and the Damping of MHD Modes in CART, Bull. Am. Phys. Soc. 36 (1991) 2309.

R.O. Dendy (Culham Laboratory) and R.W. Harvey, On the Universal Toroidal Ohm's Law for Tokamaks, General Atomics Report GA-A20343 (Decernber 1991), submitted to Physics of Fluids $B$.

R.R. Dominguez and G.M. Staebler, Impurity Induced Thermal Pinches and Anomalous Impurity Transport, Bull. Am. Phys. Soc. 36 (1991) 2344.

R.K. Fisher, J.M. McChesney, P.B. Parks, J.A. Snipes (MIT), J.L. Terry (MIT), E.S. Marmar (MIT), S.J. Zweben (PPPL), and S.S. Medley (PPPL), Impurity Pellet Alpha Diagnostic Experiments on TFTR, Bull. Am. Phys. Soc. 36 (1991) 2452.

R.W. Harvey, S.C. Chiu, M.G. McCoy (NERSC), G.D. Smith (NERSC), and T.K. Mau (UCLA), Bull. Am. Phys. Soc. 36 (1991) 2340.

R.W. Harvey, S.C. Chiu, M.G. McCoy (NERSC), G.D. Kerbel (NERSC), G.R. Smith (LLNL), and T.K. Mau (UCLA), 3D Fokker-Planck Calculation of Combined Fast Wave/Lower Hybrid and Electron Cyclotron Current Drive in Tokamaks, General Atomics Report GA-A20752 (December 1991), presented at the IAEA Technical Committee Meetirg on Fast Wave Current Drive in Reactor Scale Tokamaks, September 1991, Arles, France, and to be printed in the Proceedings.

F.J. Helton, J.M. Greene, and T.S. Taylor, Design of Feedback Control Systems, Bull. Am. Phys. Soc. 36 (1991) 2478.

F.L. Hinton and J.C. Wiley (UT), Particle and Energy Confinement Bifurcation in Tokamaks, Bull. Am. Phys. Soc. 36 (1991) 2475.

O.T. Kingsbury and R.E. Waltz, 2D-Numerical Simulation of Trapped Ion Turbulence in a Tokamak, Bull. Am. Phys. Soc. 362279 (1991). 
R.L. Miller, V.S. Chan, and T. Ohkawa, Minimum Energy Toroidal Pinch Equilibria with F'inite Pressure, Bull. Am. Phys. Soc. 36 (1991) 2308.

G.M. Staebler and R.R. Dominguez, Turbulent Viscosity Bifurcation and the H-Mode, General Atomics Report GA-A20629 (November 1991), submitted to Nuclear Fusion.

G.M. Staebler and R.R. Dominguez, Drift Wave Transport Reduction by Electric Field Profile Control, Bull. Am. Phys. Soc. 36 (1991) 2344.

A.D. Turnbull, M.S. Chu, J.M. Greene, M.S. Chance, E.J. Strait, W.W. Heidbrink (UCI), and T.S. Taylor, The Shear Alfvén Spectrum in Tokamak Geometry, Bull. Am. Phys. Sor: 36 (199i) 2377.

R.E. Waltz, J.C. DeBoo, and T. H. Osborne, Modeling Almost Dimensionally Similar Discharges, General Atomics Report GA-A20705, submitted to Nuclear Fusion.

R.E. Waltz, R.R. Dominguez, and G.W. Hammett (PPPL), Gyro-Landau Fluid Models for Toroidal Geometry, General Atomics Report GA-A20736, submitted to Physics of Fluids B.

R.E. Waltz, G.D. Kerbel (NERSC), G.W. Hammett (PPPL), and R.R. Dominguez, Simulation of Turbulence with Gyro-Landau Fluid Models, Bull. Am. Phys. Soc. 36 (1991) 2279. 


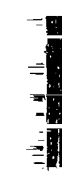

